\title{
Ferulic acid attenuates liver fibrosis and hepatic stellate cell activation via inhibition of TGF- $\beta /$ Smad signaling pathway
}

This article was published in the following Dove Press journal: Drug Design, Development and Therapy

\author{
Mao $\mathrm{Mu}^{1, *}$ \\ Shi Zuo,* \\ Rong-Min $\mathrm{Wu}^{3}$ \\ Kai-Sheng Deng' \\ Shuang Lu' \\ Juan-Juan Zhu' \\ Gao-Liang Zou' \\ Jing Yang' \\ Ming-Liang Cheng' \\ Xue-Ke Zhao' \\ 'Department of Infectious Diseases, \\ The Affiliated Hospital of Guizhou \\ Medical University, Guiyang, Guizhou, \\ China; ${ }^{2}$ Department of Hepatobiliary \\ Surgery, The Affiliated Hospital of \\ Guizhou Medical University, Guiyang, \\ Guizhou, China; ${ }^{3}$ Department of \\ Ultrasonography, The Maternity \\ Hospital of Guizhou, Guiyang, \\ Guizhou, China \\ *These authors contributed equally \\ to this work
}

Purpose: Liver fibrosis is a worldwide health issue. Development of effective new drugs for treatment of this disease is of great importance. This study investigated the therapeutic effects of ferulic acid on liver fibrosis in vitro and in vivo.

Materials and methods: Human hepatic stellate cell line (HSC) LX-2 was used for in vitro assays. Transforming growth factor $\beta 1$ (TGF- $\beta 1$ ) was used to induce hepatic fibrosis in LX-2 cells. Western blot was used to detect protein levels of collagen I, fibronectin, $\alpha$-smooth muscle actin (SMA), p-Smad2, p-Smad3, p-p38, and p-JNK. Gene expression was measured by RT-qPCR. Fluorescence staining was used to determine localization of Smad4. CCl4-induced hepatic fibrosis in SD rats was used as an in vivo model. Histological features were detected by hematoxylin and eosin staining. Levels of alanine aminotransferase (ALT), aspartate aminotransferase (AST), hexadecenoic acid (HA), and hydroxyproline (Hyp) were measured by ELISA. Results: TGF- $\beta 1$ treatment significantly increased levels of collagen I, fibronectin, $\alpha$-SMA, p-Smad2, p-Smad3, and Smad4 in LX-2 cells. Ferulic acid improved TGF- $\beta 1$-induced hepatic fibrosis via regulation of the TGF- $\beta 1 / \mathrm{Smad}$ pathway. Consistent with in vitro data, $\mathrm{CCl} 4$ caused severe hepatic fibrosis in SD rats, as determined by ALT, AST, HA, and Hyp upregulation. Protein levels of $\mathrm{p}-\mathrm{Smad} 2$ and $\mathrm{p}$-Smad3 in liver tissues were significantly increased following treatment with $\mathrm{CCl} 4$. All CCL4-induced changes were markedly attenuated by ferulic acid treatment

Conclusion: Ferulic acid potently improved hepatic fibrosis via inhibition of the TGF- $\beta 1 / \mathrm{Smad}$ pathway in vitro and in vivo. These findings provided evidence for potential use of ferulic acid to treat or prevent liver fibrosis.

Keywords: ferulic acid, TGF- $\beta 1, \mathrm{CCl}$, hepatic fibrosis, Smad signaling pathway

\section{Introduction}

Liver fibrosis is a result of wound-healing after repeated injury resulting from chronic hepatitis, cholestasis, alcohol, or drugs. ${ }^{1}$ In the damaged liver, parenchymal cells regenerate and hepatocytes are replaced by degenerative cells. ${ }^{1}$ Liver fibrosis represents the final common pathway of all chronic liver diseases and eventually leads to cirrhosis, which is end-stage liver disease. ${ }^{1}$

Hepatic stellate cells (HSCs) are key factors in the development of liver fibrosis. HSCs are normally quiescent but are activated in response to liver injury. Once activated, HSCs become proliferative and fibrogenic and accumulate extracellular matrix (ECM). ${ }^{2-4}$ Transforming growth factor- $\beta 1$ (TGF- $\beta 1$ ) is a key activator of HSCs. It acts in an autocrine or paracrine manner and is produced by Kupffer cells, sinusoidal endothelial cells, and hepatocytes. ${ }^{5,6}$ Furthermore, TGF- $\beta 1$ upregulates synthesis
Correspondence: Jing Yang; Xue-Ke Zhao Department of Infectious Diseases, The Affiliated Hospital of Guizhou Medical University, No 28, Guiyi Street, Guiyang, Guizhou 550004, China Email jingyang_1981@।26.com; xueke.zhao@yandex.com 
of proteins associated with ECM and cellular receptors of several matrix proteins. ${ }^{5-7}$ Increased ECM component expression, primarily collagen, is characteristic of hepatic fibrosis. Liver parenchymal cell destruction and fibrous scarring also occur with hepatic fibrosis. ${ }^{89}$ While hepatic fibrosis is reversible, ${ }^{10}$ the resulting structural damage to liver lobules and vasculature leads to cirrhosis, which is irreversible. Therefore, prevention and reversal of hepatic fibrosis is necessary to treat various chronic liver diseases and to prevent cirrhosis.

Ferulic acid, a derivative of cinnamic acid, has therapeutic activity against a variety of diseases. ${ }^{11,12}$ Ferulic acid is also an effective ingredient in some traditional Chinese medicines, such as Angelica sinensis. ${ }^{12}$ In addition, ferulic acid may protect against vascular endothelial dysfunction in diabetic rats when combined with astragaloside IV through regulation of the nuclear factor- $\kappa \mathrm{B}(\mathrm{NF}-\kappa \mathrm{B})$ pathway. ${ }^{12}$ Antioxidant effects of ferulic acid have also been demonstrated in previous studies. ${ }^{12-14}$ Hepatoprotective effects of ferulic acid have been demonstrated in studies using diosbulbin B and carbon tetrachloride (CCl4) as initiators of liver fibrosis. ${ }^{15-17}$

Although the protective mechanisms of ferulic acid on many diseases have been studied, few reports have studied the effects of ferulic acid on liver fibrosis. Of the studies performed, the majority have focused on the ability of ferulic acid to reduce oxidative damage. Therefore, the mechanisms underlying the anti-fibrosis effects of ferulic acid were investigated in the current study.

\section{Materials and methods}

\section{Materials}

Ferulic acid, TGF- $\beta 1$, and CCl4 were purchased from Sigma-Aldrich Co. (St Louis, MO, USA). Anti-collagen I, anti-fibronectin, anti- $\alpha$-SMA, anti-p-p38, anti-p-JNK, antip-Smad2, and anti-p-Smad3 antibodies were all obtained from Cell Signaling Technologies (CST). Commercial kits used to measure ALT, AST, HA, and Hyp were all purchased from Nanjing Jiancheng Bioengineering Institute (Nanjing, China). Other reagents, such as Dulbecco's Modified Eagle's Medium (DMEM), were purchased from Thermo Fisher Scientific (Waltham, MA, USA).

\section{Cell culture}

LX-2 human HSCs were obtained from Merck Millipore (Billerica, MA, USA) and were cultured in DMEM supplemented with $2 \%$ fetal calf serum (FCS). Cells used in experiments were subjected to not more than 10 cell passages; $5 \mathrm{ng} / \mathrm{mL}$
TGF- $\beta 1$ was used to induce target gene expression in LX-2 HSC. Exponentially growing cells were treated with TGF- $\beta 1$ for 24 hours. Dimethyl sulfoxide (DMSO) was chosen as a vehicle to dissolve ferulic acid. The DMSO solution of ferulic acid was added to the culture medium 12 hours prior to addition of TGF- $\beta 1$. $0.5 \%$ DMSO (DMEM solution) was used as control.

\section{Cell viability determination}

CCK-8 assay was used to measure LX-2 cell viability. Cells were plated at $5 \times 10^{3}$ cells per well in 96-well plates and exposed to different concentrations of ferulic acid for 24 hours. Then, the cells were incubated with $10 \mu \mathrm{L} \mathrm{CCK}-8$ for another 1 hour at $37^{\circ} \mathrm{C}$. The resulting product was measured at $450 \mathrm{~nm}$ using a microplate reader (Thermo Fisher Scientific).

\section{Immunocytochemistry}

$4 \times 10^{4}$ LX-2 HSC cells were plated on coverslips, placed in 6-well dishes and incubated for 24 hours at $37^{\circ} \mathrm{C}$. Cells were fixed in 4\% paraformaldehyde in phosphate-buffered saline (PBS) and permeabilized in $0.1 \%$ sodium citrate $/ 0.1 \%$ Triton X-100 for 15 minutes. Then the cells were incubated with primary antibodies against Smad4 (Abcam, Cambridge, MA), USA at $4{ }^{\circ} \mathrm{C}$ overnight. Antibodies were applied in PBS containing $1 \%$ bovine serum albumin, followed by incubation with secondary antibodies (Thermo Fisher Scientific) for 1 hour at $37^{\circ} \mathrm{C}$. Nuclei were stained with $4^{\prime}$, 6-diamidino-2-phenylindole (DAPI) for 15 minutes at $37^{\circ} \mathrm{C}$.

\section{Western blot assay}

Liver tissues or cells were lysed with RIPA buffer containing a protease inhibitor cocktail. Lysates were centrifuged at $10,000 \mathrm{~g}$ for 10 minutes at $4^{\circ} \mathrm{C}$. The supernatant was collected for further analysis. Total protein was measured using BCA assay kit (Pierce, Carlsbad, CA, USA). Equal amounts of total protein were loaded onto a 10\% SDS-PAGE gel and transferred onto polyvinylidene fluoride (PVDF) membranes using a wet transfer device. After blocking with 5\% non-fat milk at room temperature for 1 hour, membranes were incubated with primary antibodies overnight at $4^{\circ} \mathrm{C}$. Membranes were then incubated with the appropriate horseradish peroxidase-conjugated secondary antibody for 2 hours at room temperature. PVDF membranes were incubated with electrochemiluminescence reagent in order to visualize bands. Collagen I, fibronectin, $\alpha$-SMA, p-smad2, p-smad3, p-p38, p-JNK, and $\beta$-actin primary antibodies were 
provided by Abcam. All values were normalized to $\beta$-actin. All samples were analyzed in triplicate.

\section{RT-qPCR}

Trizol reagent (Thermo Fisher Scientific) was used to isolate total RNA from LX-2 cells. A reverse transcriptase polymerase chain reaction kit (RT-PCR) was used to reversetranscribe RNA $(2 \mu \mathrm{g})$ to obtain cDNA. A NanoDrop 8,000 spectrophotometer was used to quantify the RNA. PCR cycling procedures were as follows: $25^{\circ} \mathrm{C}$ for 5 minutes, $37^{\circ} \mathrm{C}$ for 60 minutes, and $70^{\circ} \mathrm{C}$ for 5 minutes. A 7500 real-time PCR detection system from Thermo Fisher Scientific was used in this experiment. Primers were purchased from Sangon Biotech (Shanghai, China). Collagen I: forward, 5'-TGGCCAAGAAGACATCCCTGAAGT-3'; reverse, 5'-ACATCAGGTTTCCACGTCTCACCA-3'. Fibronectin: forward, 5'-CCATCGCAA ACCGCTGCCAT-3'; reverse, 5'-AACACTTCTCAGCTATGGGCTT-3'. $\alpha$-smooth muscle actin ( $\alpha$-SMA): forward, $5^{\prime}$-ACTG AGCGTGGCTATTCCTCCGTT-3'; reverse, 5'-GCAGTGGC CATCTCATTTTCA-3'. GAPDH: forward, $5^{\prime}$-AAGAAGG TGGTGAAGCAGGC-3'; reverse, 5'-TCCACCACCCT GTTGCTGTA-3'. Target mRNA was normalized to GAPDH. GAPDH served as an endogenous control. Relative expression changes were determined using the $2^{-\Delta \Delta \mathrm{Ct}}$ method.

\section{Animal studies}

Male Wistar rats $(180 \pm 20 \mathrm{~g})$ were provided by Vital River (Beijing, China). Animals were randomly divided into four groups (six rats per group): control, $\mathrm{CCl} 4$, ferulic acid, and ferulic acid+ CCl4 group. Animals in all groups were provided a standard commercial rodent diet and were maintained on a 12-hour light/dark cycle at constant temperature and humidity. All experimental procedures were approved by the Ethical Committee of The Hospital Affiliated to Guizhou Medical University. In addition, the National Institutes of Health guide for the care and use of laboratory animals was strictly followed.

Rats in the $\mathrm{CCl} 4$ group or ferulic acid $+\mathrm{CCl} 4$ group were treated intraperitoneally (ip) with $\mathrm{CCl} 4$ dissolved in olive oil $(20 \% \mathrm{v} / \mathrm{v}, 2 \mathrm{~mL} / \mathrm{kg})$ twice per week for 8 weeks. Following this 8 -week treatment course, rats in the ferulic acid group and ferulic acid+ CCl4 group were ip dosed with $10 \mathrm{mg} / \mathrm{kg}$ ferulic acid three times per week for 8 weeks. Animals in the control group were treated with $0.9 \%$ saline three times per week for 8 weeks. Animals were sacrificed 24 hours after the last dose of ferulic acid or $0.9 \%$ saline using $\mathrm{CO}_{2}$.
Rat liver and serum samples were collected for biochemical and molecular analyses.

\section{Histopathology}

Rat liver samples were preserved in 4\% paraformaldehyde solution to obtain histological slices. After fixation, all tissues were processed, paraplast-fixed blocks sectioned ( $5 \mu \mathrm{m}$ thickness), and slides stained with hematoxylin and eosin (H\&E). Representative microphotographs were obtained using a light microscope (Nikon, Japan).

\section{Detection of ALT, AST, HA, and Hyp}

Serum levels of aspartate aminotransferase (AST) and aminotransferase (ALT) in rats were measured using ELISA kits (Nanjing Jiancheng Bioengineering Institute, Nanjing China) according to the manufacturer's protocol. An appropriate amount of liver was homogenized in $0.9 \%$ saline. The $0.9 \%$ saline homogenization suspension was used to assay liver HA and Hyp using standard commercial kits (Nanjing Jiancheng Bioengineering Institute, Nanjing, China) according to the manufacturer's instructions.

\section{Statistical analyses}

All values are presented as mean $\pm \mathrm{SD}$. Student's $t$-test was used to compare values between two groups. One-way ANOVA analysis followed by Dunnett's test was used to compare the values obtained from multiple groups. Differences were considered statistically significant at $P<0.05$.

\section{Results \\ Expression of collagen I, fibronectin, and $\alpha$-SMA was significantly upregulated by TGF- $\beta$ I in LX-2 cells}

LX- 2 cells were treated with TGF- $\beta 1$ for 24 hours, and gene expression of collagen I, fibronectin, and $\alpha$-SMA in cells was measured by RT-qPCR. As shown in Figure 1A, gene expressions of collagen I, fibronectin, and $\alpha$-SMA were notably increased by TGF- $\beta 1$ in LX- 2 cells compared with the control group. In addition, these three proteins were detected by Western blot. The original image and quantification results are summarized in Figure 1B-F. Consistent with RT-qPCR data, protein expressions of collagen I, fibronectin, and $\alpha$-SMA in LX-2 HSC were significantly increased by TGF- $\beta 1$. All these data indicated that the in vitro liver fibrosis model was successfully established. 
A<smiles>COc1cc(/C=C/C(=O)O)ccc1O</smiles>

Structure of ferulic acid

D

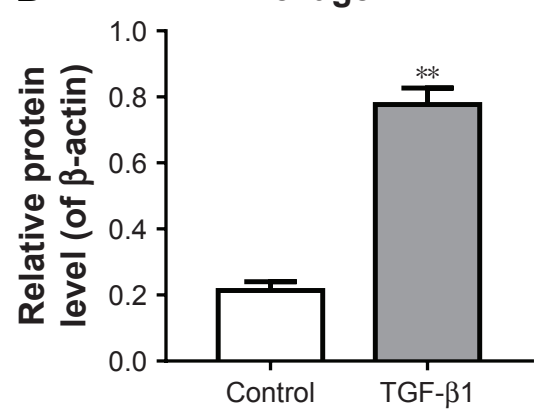

B

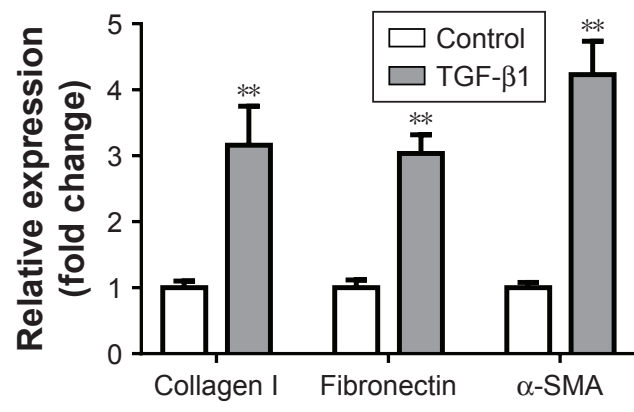

C

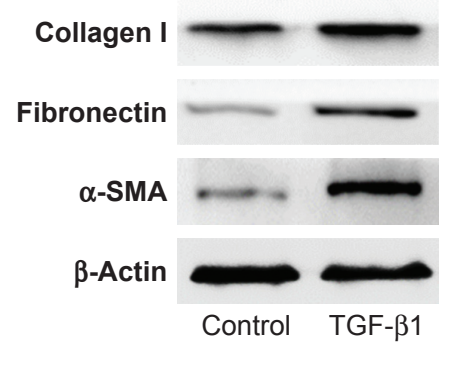

E

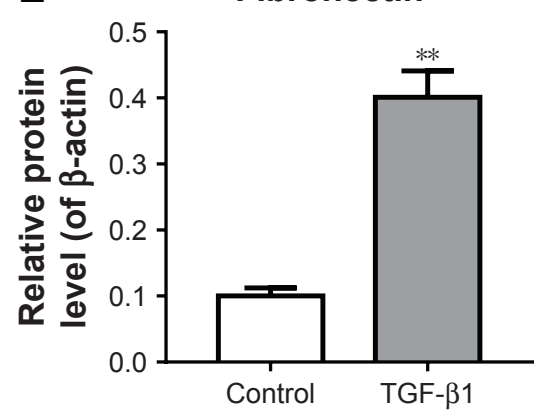

$\alpha-S M A$

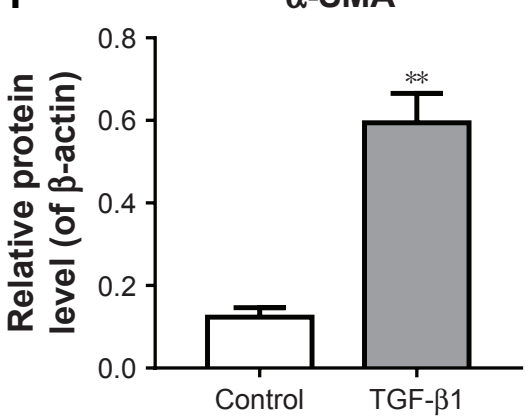

Figure I The expression of collagen I, fibronectin, and $\alpha$-SMA were significantly upregulated by TGF- $\beta$ I in LX- 2 cells. Exponentially growing LX-2 cells were treated with $5 \mathrm{ng} / \mathrm{mL}$ TGF- $\beta$ I for 24 hours. (A) The chemical structure of ferulic acid. (B) Collagen I, fibronectin, and $\alpha$-SMA gene levels in the control or TGF- $\beta$ I group were detected by RT-qPCR. (C) The representative Western blot image of collagen I, fibronectin, and $\alpha$-SMA in the control group or TGF- $\beta$ I group. (D-F) The quantification of collagen I, fibronectin, and $\alpha$-SMA proteins expressions in LX-2 cells.

Notes: $\mathrm{n}=3$ in each group. $* * \mathrm{p}<0.01$ vs control group.

Abbreviations: $\alpha$-SMA, $\alpha$-smooth muscle actin; TGF- $\beta$ I, transforming growth factor- $\beta$ I.

\section{Ferulic acid improved TGF- $\beta$ I-induced in vitro liver fibrosis}

We next evaluated the effects of ferulic acid on TGF- $\beta 1$ induced in vitro liver fibrosis. The chemical structure of ferulic acid is shown in Figure 2A. CCK-8 results indicated that $50 \mu \mathrm{M}$ ferulic acid was cytotoxic to LX-2 cells (Figure 2B). Based on this data, $30 \mu \mathrm{M}$ ferulic acid was used for subsequent in vitro assays. As shown in Figure $2 \mathrm{C}-\mathrm{F}$, TGF- $\beta 1$ induced collagen I, fibronectin, and $\alpha$-SMA upregulation were significantly reversed by ferulic acid. However, ferulic acid alone had no effect on these proteins. These results suggested that TGF- $\beta 1$-induced liver fibrosis in LX-2 cells could be improved by ferulic acid.

\section{Ferulic acid attenuated TGF- $\beta$ I-induced in vitro liver fibrosis via inhibition of the TGF- $\beta$ I/Smad pathway}

We next investigated the effects of ferulic acid on TGF- $\beta 1 /$ Smad, p38, and JNK pathways in LX-2 cells. As indicated in Figure $3 \mathrm{~A}-\mathrm{C}$, $\mathrm{p}-\mathrm{Smad} 2$ and $\mathrm{p}-\mathrm{Smad} 3$ were significantly increased by TGF- $\beta 1$ treatment. These increases were markedly attenuated by ferulic acid. In contrast, p38 and JNK pathways were not to be involved in TGF- $\beta 1$-induced in vitro liver fibrosis (Figure 3A, D, and E). Ferulic acid alone had limited effects on these proteins. Smad4 is typically localized in the cytoplasm. Following activation of $\mathrm{Smad} 2$ and $\mathrm{Smad} 3$, these three proteins form a complex that translocates to the nucleus. In the present study, Smad4 was localized primarily in the cytoplasm in the control and ferulic acid groups. However, TGF- $\beta 1$ significantly induced localization of Smad4 in the nucleus. Ferulic acid reversed TGF- $\beta 1$-induced Smad4 nuclear translocation (Figure 3F). These data show that ferulic acid attenuated TGF- $\beta 1$-induced in vitro liver fibrosis via inhibition of the TGF- $\beta 1 /$ Smad pathway.

\section{Ferulic acid improved $\mathrm{CCl} 4$-induced rat liver fibrosis by inhibition of the Smad pathway}

To further confirm the anti-fibrosis effects of ferulic acid, an in vivo rat liver fibrosis model was established using CC14. As shown in Figure 4A, inflammatory cell infiltration, fragmented hepatic nuclei, and formation of collagenous fibers were observed in the CCl4-treated group. Damage caused by $\mathrm{CCl} 4$ was significantly attenuated by ferulic acid, 
A

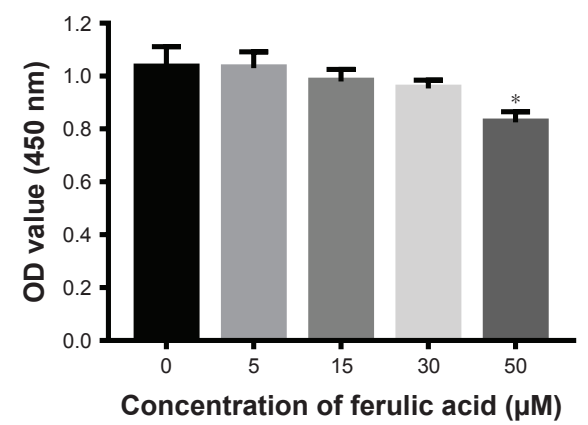

B

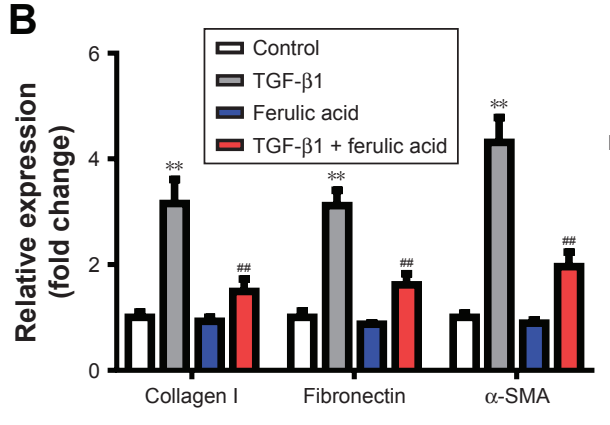

C

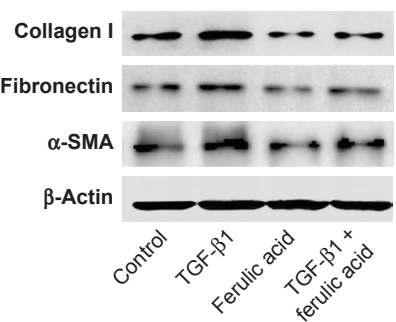

D

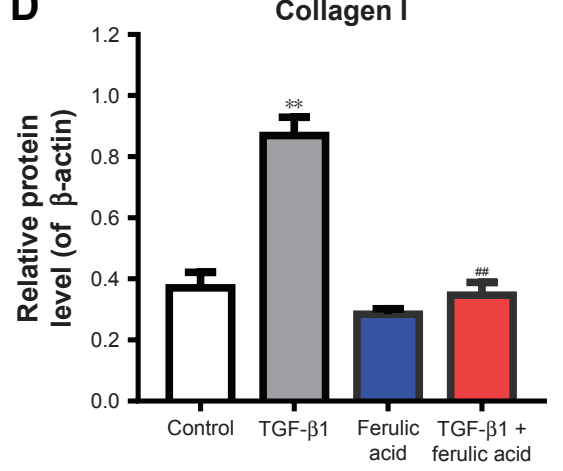

E

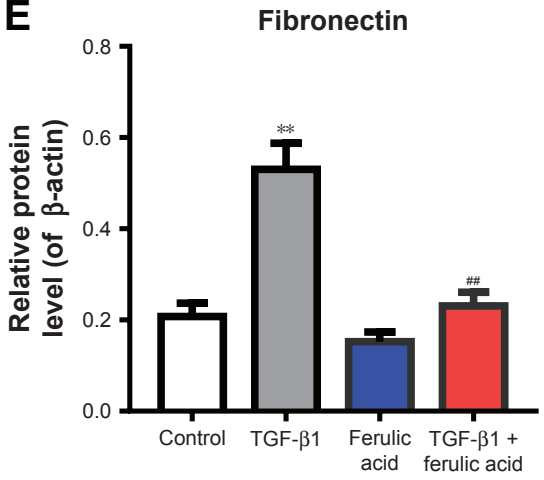

$\mathbf{F}$
$\alpha-S M A$

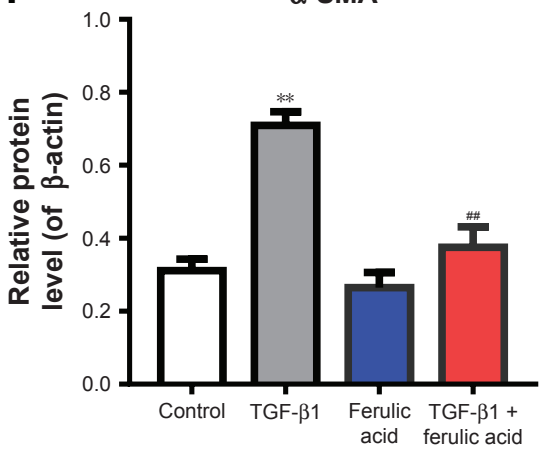

Figure 2 Ferulic acid improved TGF- $\beta$ I-induced in vitro liver fibrosis. (A) LX-2 cells were treated with indicated concentrations of ferulic acid for 24 hours and cell viability was detected with CCK-8 kit. (B) A density of $5 \times 103$ cells/well was planted in 96 -well plate and exposed to ferulic acid for I 2 hours before TGF- $\beta$ I treatment. Collagen I, fibronectin, and $\alpha$-SMA gene levels in control, TGF- $\beta$ I, ferulic acid group, TGF- $\beta$ I plus ferulic acid groups were detected with RT-qPCR. (C) The representative Western blot image of collagen I, fibronectin, and $\alpha$-SMA in control, TGF- $\beta$ I, ferulic acid, or TGF- $\beta$ I plus ferulic acid group. (D-F) The quantification of collagen I, fibronectin, and $\alpha$-SMA proteins expressions in LX-2 cells. The relative gene expression of GAPDH was used as the internal standard in RT-qPCR assay. The quantification results of the Western blot was normalized to the expression of $\beta$-actin by Image Pro Plus.

Notes: $n=3$ in each group. ${ }^{*} P<0.05$ vs control group; $* * P<0.01$ vs control group, ${ }^{\#} P<0.0$ I vs TGF- $\beta$ I group.

Abbreviations: $\alpha$-SMA, $\alpha$-smooth muscle actin; TGF- $\beta$ I, transforming growth factor- $\beta$ I.

while ferulic acid alone did not influence these biochemical markers. In addition, the levels of ALT and AST in plasma, and HA and Hyp in liver tissues, were upregulated by $\mathrm{CCl} 4$ (Figure 4B-E). Ferulic acid attenuated expression of these biomarkers (Figure 4B-E). Consistent with in vitro results, protein levels of $\mathrm{p}$-Smad2 and p-Smad3 were significantly upregulated by $\mathrm{CCl} 4$ in liver tissues, but returned to normal as a result of ferulic acid administration (Figure 5A-C). These results demonstrated that ferulic acid could improve CCl4-induced rat liver fibrosis by inhibition of the Smad pathway (Figure 5D).

\section{Discussion}

Excessive accumulation of ECM proteins, including collagen, occurs in most types of chronic liver diseases, and can induce liver fibrosis. ${ }^{18}$ Advanced stages of liver fibrosis can lead to liver cirrhosis and failure. ${ }^{18}$ Despite increased understanding of the cellular and molecular mechanisms underlying liver fibrosis, many antifibrotic therapies are still under investigation.
Ferulic acid, a phenolic acid, has been reported to exert beneficial medicinal effects. ${ }^{11,12}$ However, few studies have examined the effect of ferulic acid on hepatocyte damage. In the present study, the mechanisms underlying antifibrotic effects of ferulic acid in vitro and in vivo were investigated. The results indicated that expression of $\alpha$-SMA, a marker of HSC activation, was significantly increased by exogenous TGF- $\beta 1$. Ferulic acid inhibited TGF- $\beta 1$-induced elevation of $\alpha$-SMA, indicating that HSC activation might be prevented by ferulic acid. Furthermore, ferulic acid significantly reduced fibronectin expression in TGF- $\beta 1$-treated LX-2 cells.

TGF- $\beta 1$, the principal transforming growth factor isoform, is implicated in liver fibrosis. ${ }^{5,6}$ TGF- $\beta 1$ is bound to a latency-associated peptide and is, thus, maintained in an inactive state. Once activated, TGF- $\beta 1$ exerts its biological and pathological activities via the Smad signaling pathways. ${ }^{19}$ Smad signal transduction pathways mediate TGF- $\beta 1$-induced collagen synthesis and play a crucial role in development of liver fibrosis or liver recovery. ${ }^{19}$ After phosphorylation, the Smad 2/3 complex binds with Smad 4. 
A

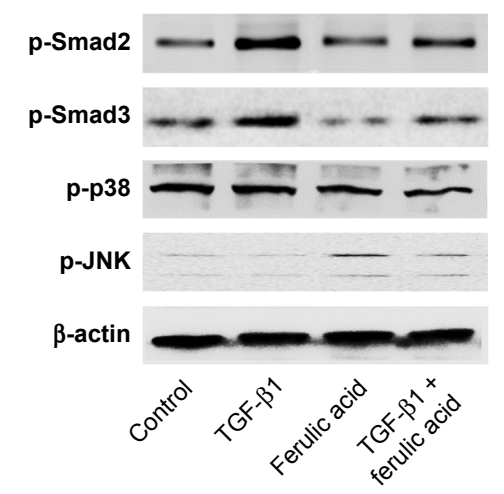

D

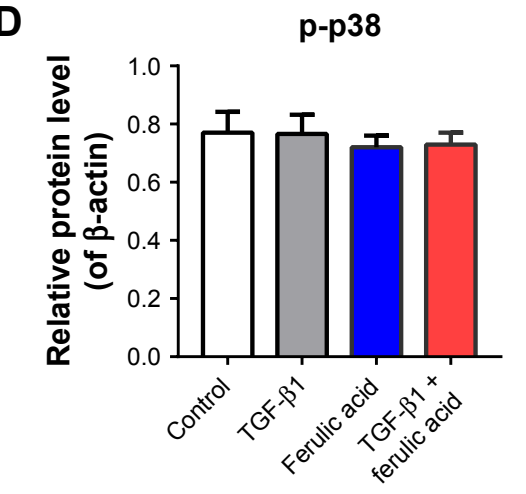

E

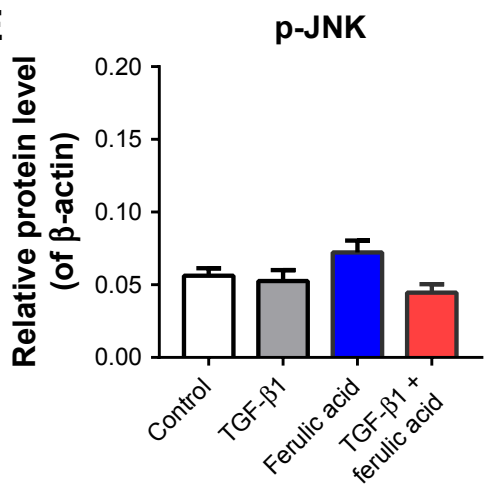

B

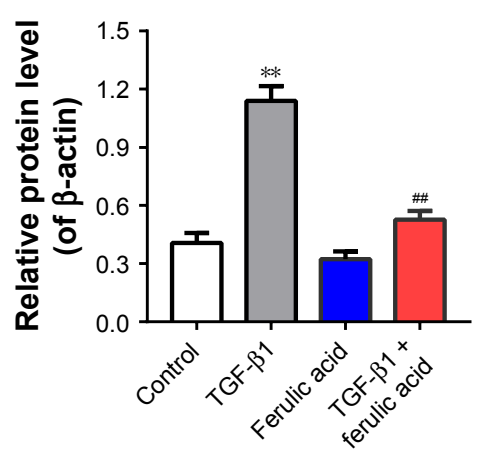

F
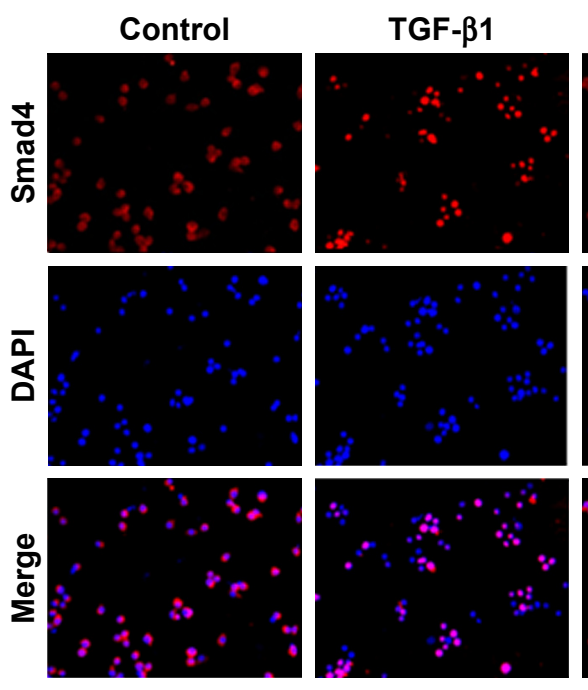
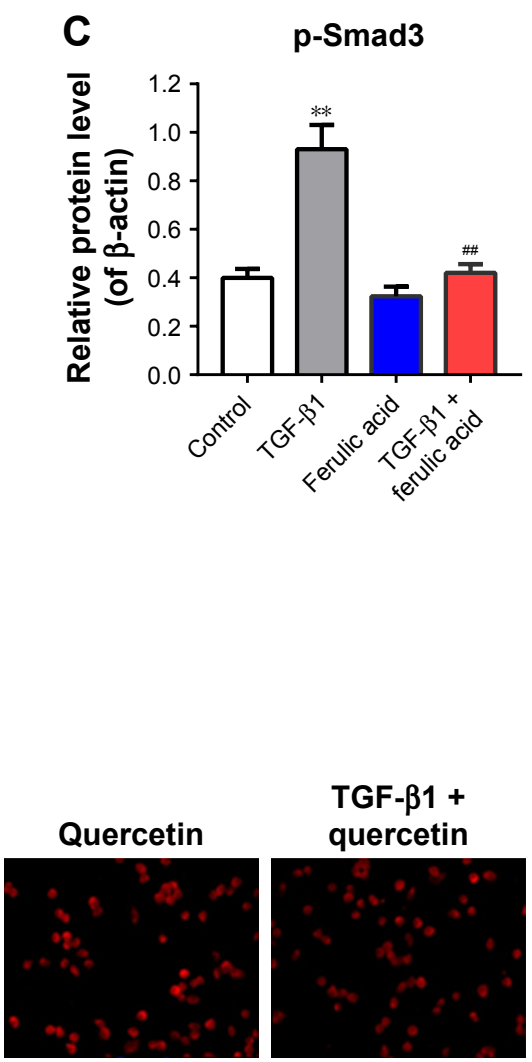

TGF- $\beta 1+$

quercetin
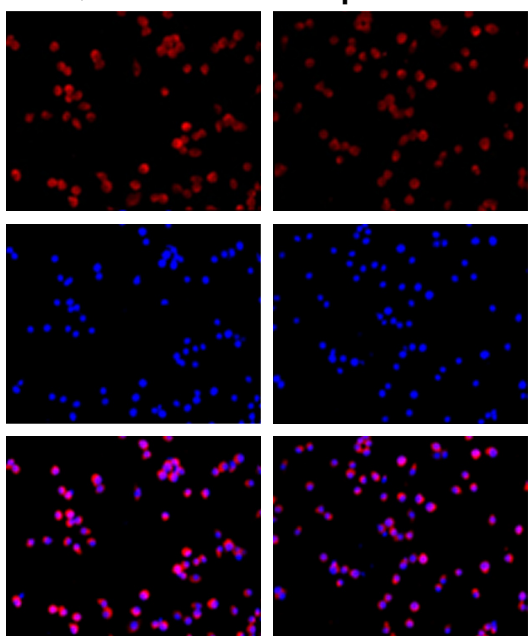

Figure 3 Ferulic acid attenuated TGF- $\beta$ I-induced liver fibrosis in LX-2 cells via inhibition of the TGF- $\beta$ I/Smad pathway. (A) The representative Western blot image of p-Smad2, p-Smad3, p-p38, and p-JNK in LX-2 cells treated with TGF- $\beta$ I and/or ferulic acid. (B-E) The quantification of proteins p-Smad2, p-Smad3, p-p38, and p-JNK in LX-2 cells by normalizing to the expression of $\beta$-actin with Image Pro Plus. (F) The representative image of cell location of Smad 4 in LX-2 by immunocytochemistry and DAPI staining (magnification $\times 200$ ).

Notes: Nuclei were stained with DAPI for 15 minutes at $37^{\circ} \mathrm{C} ; \mathrm{n}=3$ in each group. ${ }^{* *} P<0.01$ vs control group, $P<0.0$ I vs TGF- $\beta$ I group. Abbreviations: DAPI, 4', 6-diamidino-2-phenylindole; TGF- $\beta$ I, transforming growth factor- $\beta$ I.

This translocates to the nucleus to regulate expression of target genes. ${ }^{19}$ To elucidate the relationship of TGF- $\beta / \mathrm{Smad}$ signaling with liver fibrosis, the mechanisms by which ferulic acid attenuated TGF- $\beta 1$-induced LX- 2 cell fibrosis were investigated. Our study showed that phosphorylation of Smad $2 / 3$ was downregulated after ferulic acid treatment. Ferulic acid further inhibited translocation of Smad 4 to the nucleus. These results indicated that inhibition of TGF- $\beta /$ Smad signaling may be a key mechanism by which ferulic acid exerted its antifibrotic effects on TGF- $\beta 1$-treated LX-2 cells.
CCl4 has been extensively used as a model compound in rodents to induce acute liver injury, hepatic fibrosis, or cirrhosis. ${ }^{20,21}$ To further explore the anti-fibrotic effects of ferulic acid, a CCl4-induced rat liver fibrosis model was established based on the method of Domitrović and Jakovac. ${ }^{22}$ In our study, histological analysis showed that $\mathrm{CCl} 4$ caused hepatic cells necrosis and collagen matrix accumulation with formation of interconnecting complete septa, which divided the parenchyma into separate fragments in rat liver. Consistent with in vitro data, ferulic acid suppressed liver 
A
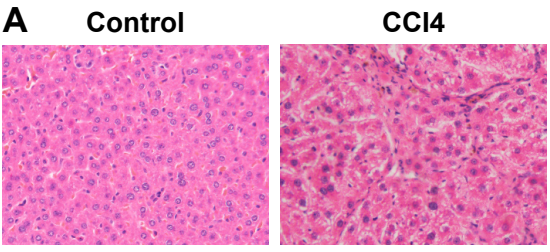

$\mathrm{CCl} 4+$

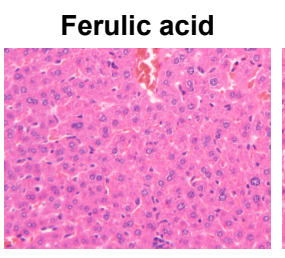

ferulic acid

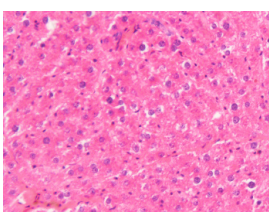

B

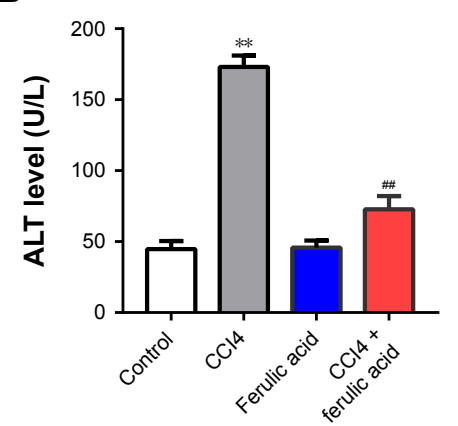

D

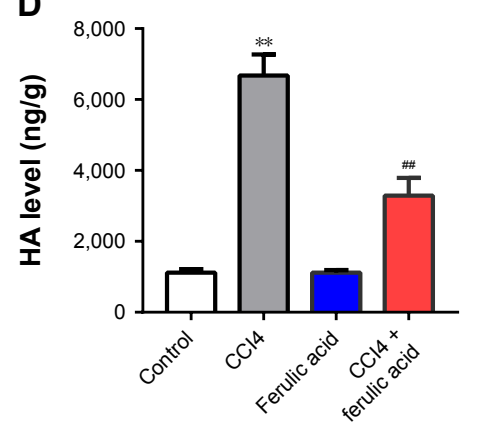

C

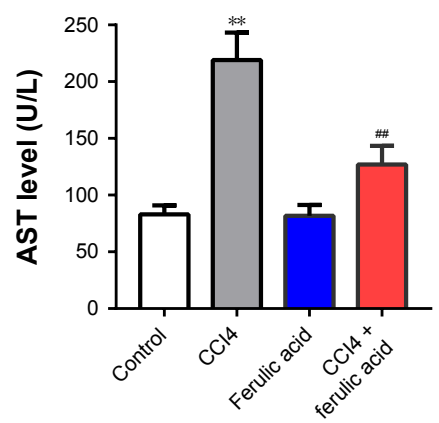

E

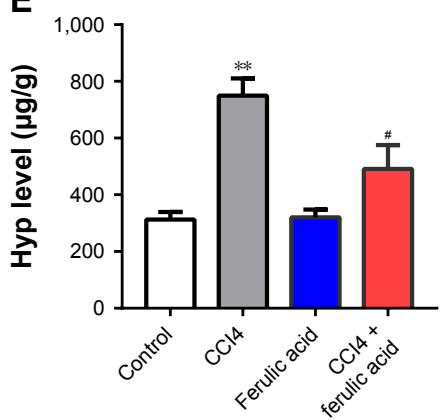

Figure 4 Ferulic acid improved CCl4-induced rat liver fibrosis. (A) The representative H\&E images of liver in control, CCl4, ferulic acid, or CCl4 plus ferulic acid groups. The images were captured under a light microscope with 200 times amplification. (B-E) Rat serums and liver tissues in each group were collected at the end of the animal experiment.

Notes: The levels of ALT, AST in serums and HA, Hyp in liver tissues were determined by using commercial available kits; $n=6$ in each group. ${ }^{* *} P<0.0 \mathrm{I}$ vs control group, ${ }^{\#} P<0.05, \# P<0.01$ vs $\mathrm{CCl} 4$ group.

Abbreviations: ALT, alanine aminotransferase; AST, aspartate aminotransferase; HA, hexadecenoic acid; H\&E, hematoxylin and eosin; Hyp, hydroxyproline.

A

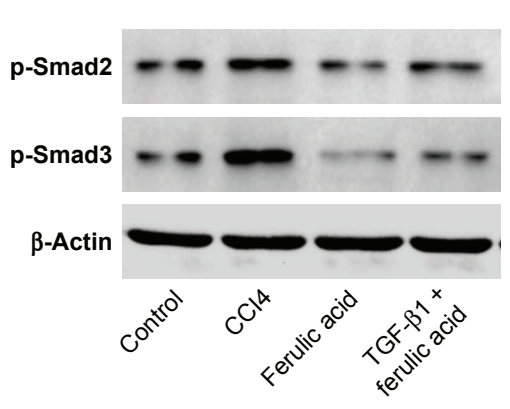

B

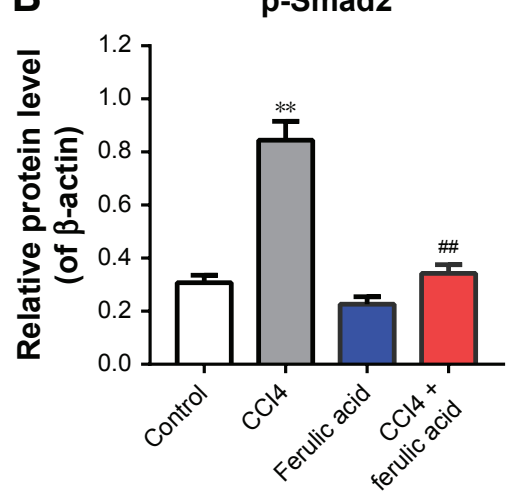

C p-Smad3

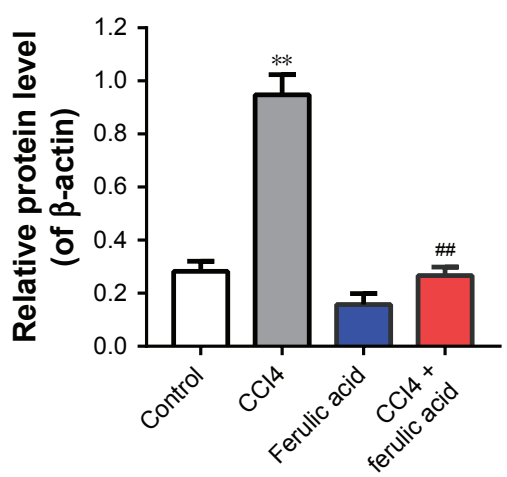

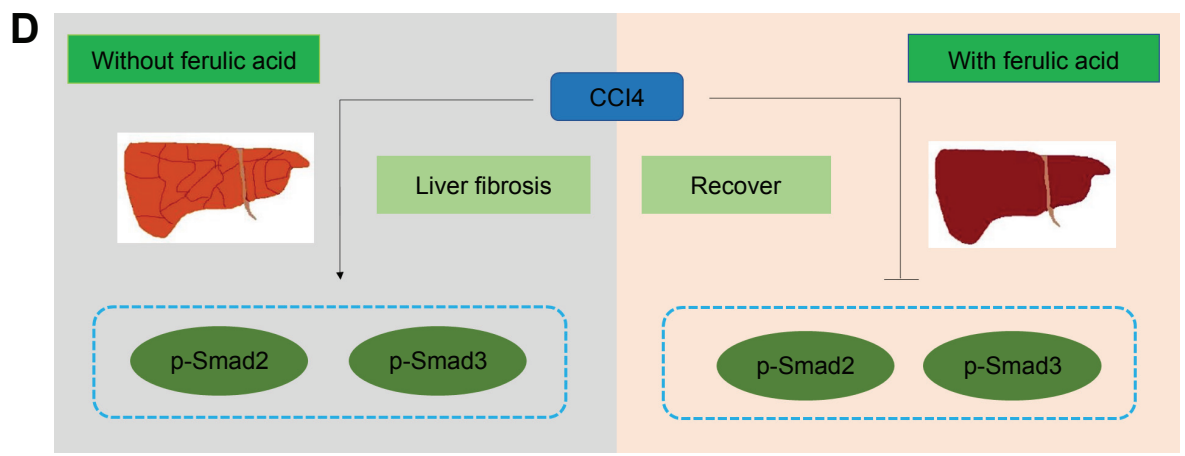

Figure 5 Ferulic acid regulated Smad pathway in CCl4-treated rat. (A) The representative Western blot image of p-Smad2 and p-Smad3 in rats treated with CCl4 and/or ferulic acid group. (B, C) The quantification of proteins $\mathrm{p}-\mathrm{Smad} 2$ and $\mathrm{p}$-Smad3 in liver tissues by normalizing to the expression of $\beta$-actin with Image Pro Plus; (D) A flow diagram to represent the molecular pathway regulated by ferulic acid in CCl4-treated rat.

Notes: $\mathrm{n}=6$ in each group. ${ }^{* *} \mathrm{P}<0.01$ vs control group, ${ }^{\#} \mathrm{P}<0.01$ vs $\mathrm{CCl} 4$ group. 
fibrogenesis by reducing the thickness of the bridging fibrotic septa. These results suggested that ferulic acid might decrease areas of hepatic fibrosis. In addition, a primary indication of hepatic damage induced by $\mathrm{CCl} 4$ was obtained by evaluating the levels of common hepatic enzymatic markers of injury such as AST and ALT. ${ }^{23}$ After treating with CCl4, levels of AST and ALT were significantly increased compared with the control group. These biomarkers enter the circulatory system due to altered permeability of membranes. Increased levels of these biomarkers reflected severe damage to the structural integrity of the liver. ${ }^{24,25}$ Administration of ferulic acid significantly attenuated $\mathrm{CCl}$-induced elevation of AST and ALT, indicating its hepatoprotective activity.

Levels of HA and Hyp in liver are also important indices reflecting the degree of liver fibrosis. ${ }^{26}$ In our study, CC14treated rats showed higher levels of HA and Hyp, with marked reduction in these markers by ferulic acid treatment, suggesting that ferulic acid treatment could attenuate $\mathrm{CCl} 4$ induced liver fibrosis.

\section{Conclusion}

We demonstrated that ferulic acid was able to attenuate TGF- $\beta 1$-induced HSCs activation by inhibiting Smad 2/3 phosphorylation and subsequent Smad 4 signal transduction. Interruption of these pathways contributed to reversal of the fibrotic process. These results suggested that ferulic acid may be useful in stopping or reversing progression of liver fibrosis and might be used in development of new therapeutic drugs for treatment of chronic liver diseases.

\section{Acknowledgment}

This work was supported by the National Natural Science Foundation of China (grant numbers 81560104 and 81860115 to Xue-Ke Zhao).

\section{Disclosure}

The authors report no conflicts of interest in this work.

\section{References}

1. Friedman SL. Liver fibrosis - from bench to bedside. J Hepatol. 2003; 38(Suppl 1):S38-S53.

2. Pinzani M, Marra F. Cytokine receptors and signaling in hepatic stellate cells. Semin Liver Dis. 2001;21(3):397-416.

3. Lotersztajn S, Julien B, Teixeira-Clerc F, Grenard P, Mallat A. Hepatic fibrosis: molecular mechanisms and drug targets. Апnи Rev Pharmacol Toxicol. 2005;45:605-628.

4. Friedman SL. Molecular regulation of hepatic fibrosis, an integrated cellular response to tissue injury. J Biol Chem. 2000;275(4):2247-2250.

5. Hernandez-Gea V, Friedman SL. Pathogenesis of liver fibrosis. Annu Rev Pathol. 2011;6:425-456.

6. Li X, Wang Y, Wang H, Huang C, Huang Y, Li J. Endoplasmic reticulum stress is the crossroads of autophagy, inflammation, and apoptosis signaling pathways and participates in liver fibrosis. Inflamm Res. 2015;64(1):1-7.
7. Cong M, Liu T, Wang P, et al. Antifibrotic effects of a recombinant adeno-associated virus carrying small interfering RNA targeting TIMP-1 in rat liver fibrosis. Am J Pathol. 2013;182(5):1607-1616.

8. Baiocchini A, Montaldo C, Conigliaro A, et al. Extracellular Matrix Molecular Remodeling in Human Liver Fibrosis Evolution. PLoS One. 2016;11(3):e0151736

9. Karsdal MA, Manon-Jensen T, Genovese F, et al. Novel insights into the function and dynamics of extracellular matrix in liver fibrosis. Am J Physiol Gastrointest Liver Physiol. 2015;308(10):G807-G830.

10. Brenner DA. Reversibility of liver fibrosis. Gastroenterol Hepatol. 2013;9(11):737-739.

11. Son MJ, Rico CW, Nam SH, Kang MY. Effect of oryzanol and ferulic acid on the glucose metabolism of mice fed with a high-fat diet. J Food Sci. 2011;76(1):H7-H10.

12. Yin Y, Qi F, Song Z, Zhang B, Teng J. Ferulic acid combined with astragaloside IV protects against vascular endothelial dysfunction in diabetic rats. Biosci Trends. 2014;8(4):217-226.

13. Jain PG, Surana SJ. Isolation, characterization and hypolipidemic activity of ferulic acid in high-fat-diet-induced hyperlipidemia in laboratory rats. EXCLI J. 2016;15:599-613.

14. Totani N, Tateishi S, Takimoto T, Shinohara R, Sasaki H. Ferulic acid esters and weight-loss promoting effects in rats. J Oleo Sci. 2012;61(6): 331-336.

15. Niu C, Sheng Y, Zhu E, Ji L, Wang Z. Ferulic acid prevents liver injury induced by Diosbulbin B and its mechanism. Biosci Trends. 2016; 10(5):386-391.

16. Wang JM, Sheng YC, Ji LL, Wang ZT. Ferulic acid prevents liver injury and increases the anti-tumor effect of diosbulbin B in vivo. $J$ Zhejiang Univ Sci B. 2014;15(6):540-547.

17. Yang C, Li L, Ma Z, et al. Hepatoprotective effect of methyl ferulic acid against carbon tetrachloride-induced acute liver injury in rats. Exp Ther Med. 2018;15(3):2228-2238.

18. Satapathy SK, Sanyal AJ. Epidemiology and natural history of nonalcoholic fatty liver disease. Semin Liver Dis. 2015;35(3):221-235.

19. Dooley S, Delvoux B, Streckert M, et al. Transforming growth factor beta signal transduction in hepatic stellate cells via Smad2/3 phosphorylation, a pathway that is abrogated during in vitro progression to myofibroblasts. TGFbeta signal transduction during transdifferentiation of hepatic stellate cells. FEBS Lett. 2001;502(1-2):4-10.

20. Domitrović R, Jakovac H, Romić Z, Rahelić D, Tadić Z. Antifibrotic activity of Taraxacum officinale root in carbon tetrachloride-induced liver damage in mice. J Ethnopharmacol. 2010;130(3):569-577.

21. Yao Q, Lin Y, Li X, Shen X, Wang J, Tu C. Curcumin ameliorates intrahepatic angiogenesis and capillarization of the sinusoids in carbon tetrachloride-induced rat liver fibrosis. Toxicol Lett. 2013;222(1):72-82.

22. Domitrović R, Jakovac H. Antifibrotic activity of anthocyanidin delphinidin in carbon tetrachloride-induced hepatotoxicity in mice. Toxicology. 2010;272(1-3):1-10.

23. Dai N, Zou Y, Zhu L, Wang HF, Dai MG. Antioxidant properties of proanthocyanidins attenuate carbon tetrachloride (CCl4)-induced steatosis and liver injury in rats via CYP2E1 regulation. $J$ Med Food. 2014;17(6):663-669.

24. Manna P, Sinha M, Sil PC. Aqueous extract of Terminalia arjuna prevents carbon tetrachloride induced hepatic and renal disorders. BMC Complement Altern Med. 2006;6:33.

25. Raja S, Ahamed KF, Kumar V, Mukherjee K, Bandyopadhyay A, Mukherjee PK. Antioxidant effect of Cytisus scoparius against carbon tetrachloride treated liver injury in rats. J Ethnopharmacol. 2007;109(1): $41-47$.

26. Li W, Wu Y, Zhu C, Wang Z, Gao R, Wu Q. Anti-fibrosis effects of Huisheng oral solution in CCl4-induced hepatic fibrosis in rat. Indian J Pharmacol. 2014;46(2):216-221. 


\section{Publish your work in this journal}

Drug Design, Development and Therapy is an international, peerreviewed open-access journal that spans the spectrum of drug design and development through to clinical applications. Clinical outcomes, patient safety, and programs for the development and effective, safe, and sustained use of medicines are the features of the journal, which has also been accepted for indexing on PubMed Central. The manuscript management system is completely online and includes a very quick and fair peer-review system, which is all easy to use. Visit http://www.dovepress.com/testimonials.php to read real quotes from published authors.

Submit your manuscript here: http://www.dovepress.com/drug-design-development-and-therapy-journal 